\title{
Anti-amphiphysin I antibodies in patients with paraneoplastic neurological disorders associated with small cell lung carcinoma
}

\author{
A Saiz, J Dalmau, M Husta Butler, Q Chen, J Y Delattre, P De Camilli, F Graus
}

Service of Neurology and Institut

d'Investigació

Biomèdica August Pi i

Sunyer (IDIBAPS),

Hospital Clínic,

University of

Barcelona, Barcelona.

Spain

A Saiz

F Graus

Department of

Neurology, Memorial

Sloan-Kettering

Cancer Center, New

York, USA

J Dalmau

Department of Cell Biology and Howard Hughes Medical

Institute, Yale

University School of

Medicine, New Haven,

Conn, USA

$M$ Husta Butler

P De Camilli

Service of Neurology,

Hôpital de la

Pitié-Salpêtrière,

INSERM U134, Paris,

France

Q Chen

J Y Delattre

Correspondence to:

Dr Francesc Graus, Servei de

Neurologia, Hospital Clínic,

Villarroel 170, Barcelona

08036. Spain. Telephone

00343 2272213; fax 00343

2275414; email

graus@medicina.ub.es

Received 9 March 1998 and in revised form

3 September 1998

Accepted 11 September

1998
Abstract

Patients with stiff man syndrome and breast cancer develop anti-amphiphysin I antibodies that primarily recognise the $C$ terminus of the protein. Anti-amphiphysin I antibodies have also been identified in a few patients with paraneoplastic neurological disorders (PND) and small cell lung cancer (SCLC). The frequency of anti-amphiphysin $I$ antibodies in patients with SCLC and PND was analysed and the epitope specificity of these antibodies was characterised.

Anti-amphiphysin I antibodies were evaluated by immunohistochemistry on human and rat cerebellum and immunoblots of rat brain homogenates. Serum samples included 134 patients with PND and anti-Hu antibodies ( $83 \%$ had SCLC), 44 with SCLC and PND without antiHu-antibodies, 63 with PND and either Yo, Ri, or Tr antibodies, 146 with SCLC without PND, and 104 with non-PND. Positive serum samples were confirmed with immunoblots of recombinant human amphiphysin I and immunoreacted with five overlapping peptide fragments covering the full length of the molecule.

Serum samples positive for antiamphiphysin I antibodies included those from seven $(2.9 \%)$ patients with PND and two (1.4\%) with SCLC without PND. Six of the seven anti-amphiphysin I antibody positive patients with PND had SCLC (three with $\mathrm{Hu}$-antibodies), and one had anti-Hu-antibodies but no detectable tumour. The PND included encephalomyelitis/sensory neuropathy (five patients), cerebellar degeneration (one), and opsoclonus (one). All anti-amphiphysin I antibodies reacted with the $C$ terminus of amphiphysin $I$, but seven also recognised other fragments of the molecule.

In conclusion, anti-amphiphysin I antibodies are present at low frequency in patients with SCLC irrespective of the presence of an associated PND. All antiamphiphysin I antibody positive serum samples have in common reactivity with the $\mathbf{C}$ terminus of the protein.

(F Neurol Neurosurg Psychiatry 1999;66:214-217)
Keywords: autoantibodies; amphiphysin I; paraneoplastic; small cell lung carcinoma; stiff man syndrome

Amphiphysin I, a nerve terminal protein with a putative role in endocytosis, ${ }^{1}$ is recognised by autoantibodies present in the serum and CSF samples from female patients with paraneoplastic stiff man syndrome (SMS) and breast cancer. ${ }^{23}$ The anti-amphiphysin I antibodies of these patients predominantly react with the $\mathrm{C}$ terminus of the protein. ${ }^{14} \mathrm{~A}$ recent study ${ }^{5}$ identified anti-amphiphysin I antibodies in the serum of three patients with small cell lung carcinoma (SCLC) and paraneoplastic encephalomyelitis and sensory neuronopathy (PEM/SN), a disorder usually associated with anti-Hu-antibodies. ${ }^{6}$ The serum of one of these patients had anti-Hu antibodies but none of them developed typical SMS. Although this study suggested that PEM/SN in patients with SCLC may associate with several autoantibody specificities and that anti-amphiphysin I antibodies are not restricted to SMS, the frequency and specificity of these associations were not defined. A few additional cases of antiamphiphysin I antibody positive patients with PND other than SMS associated with either breast or lung cancer were reported. ${ }^{7}$ To clarify these clinical-serological associations is important because these antibodies are considered good markers of specific types of paraneoplastic syndromes and tumours.

In the present study, we analysed the frequency of anti-amphiphysin I antibodies in a large series of patients with PND other than SMS, to determine whether: (1) antiamphiphysin I antibodies are associated with a particular type of PND or tumour and (2) whether amphiphysin I autoepitopes differ in patients with different clinical syndromes.

Materials and methods

Serum samples were obtained from 241 patients with PND, 146 with SCLC without PND or anti-Hu-antibodies, and 104 with non-paraneoplastic neurological disorders usually considered in the differential diagnosis of PND (cerebellar disorders 56, sensory neuropathy 41; opsoclonus seven). Among the 241 patients with PND, 134 had PEM/SN (83\% 
had SCLC), 44 had SCLC and PND without anti-Hu antibodies (paraneoplastic cerebellar degeneration 30; PEM/SN seven; opsoclonus seven), and 63 had PND and either Yo, Ri, or $\mathrm{Tr}$ antibodies. Serum samples were collected in three of the participant laboratories (Barcelona, Paris, and New York) and kept frozen at $-70^{\circ} \mathrm{C}$. Serum samples from two patients with paraneoplastic SMS and breast cancer were used as positive controls.

Immunohistochemistry on human and rat cerebellum (serum screening dilution 1:500) and immunoblot of rat brain homogeneate (serum dilution 1:10 000) were done as previously described in detail. ${ }^{89}$ To make the anti-amphiphysin I antibody detection more uniform, all immunoblots were prepared in the same laboratory (Hospital Clinic, Barcelona) and nitrocellulose strips containing the same amount of immobilised antigen were sent to the other two participating laboratories (Paris and New York) that processed the strips with the same protocol.

CRITERIA FOR THE PRESENCE OF

ANTI-AMPHIPHYSIN I ANTIBODIES

A serum was considered positive for antiamphiphysin I antibodies when immunoblots of rat brain homogenate showed a reactive band of identical electrophoretic mobility (around $128 \mathrm{kDa}$ ) than that obtained with the positive control. All positive and seven randomly taken negative serum samples were subsequently evaluated by indirect immunofluorescence on formaldehyde fixed rat cerebellar tissue $^{2}$ and immunoblots of recombinant human amphiphysin $\mathrm{I}^{4}$ at Yale University (MHB, PDC). Serum samples were tested at dilutions of 1:4 and 1:500 for the immunofluorescence and the immunoblot experiments respectively. Clinical information was blinded from investigators performing these immunoblots.

EPITOPE ANALYSIS

Glutathione S-transferase (GST) fusion proteins consisting of five overlapping fragments of amphiphysin I were prepared as described previously. ${ }^{4}$ The five GST fusion proteins were subjected to SDS-PAGE ( $10 \%$ acrylamide) and western blotting as previously described. ${ }^{10}$ Monoclonal antibodies directed to each fragment of amphiphysin I were used to identify the correct band. Positive and negative human serum samples were also used as controls.

\section{Results}

IMMUNOBLOT STUDIES

Nine serum samples immunoreacted with a band identical with that obtained with the positive control in immunoblots of rat brain homogenate (figure). In all of them, the presence of anti-amphiphysin I antibodies was confirmed in immunoblots of recombinant human amphiphysin I, whereas seven negative serum samples by the screening criteria, blindly examined with the positive ones, did not react with the recombinant protein. All nine positive samples reacted with fragment $\mathrm{V}$ of amphiphysin I that includes the $\mathrm{C}$ terminus

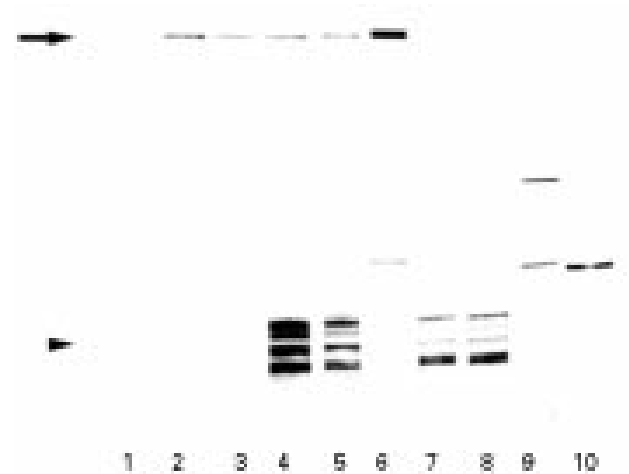

Immunoblots of rat brain homogenate probed with a normal human serum (lane 1), serum from paraneoplastic SMS and breast cancer with anti-amphiphysin I antibodies, as positive controls (lanes 2,3), PND and SCLC (lanes 4-8), and SCLC without PND (lanes 9-10). Three serum samples from patients with PND and SCLC (lanes 4-6) immunoreacted with a band (around $128 \mathrm{kDa}$ ) of the same electrophoretic mobility as that recognised by the positive anti-amphiphysin I antibody serum samples (arrow). Four serum samples also presented anti-Hu antibodies (arrowhead).

of the molecule, ${ }^{4}$ but the reactivity with the other four fragments of amphiphysin I was not uniform with seven samples recognising multiple fragments.

\section{IMMUNOHISTOCHEMISTRY STUDIES}

None of the nine positive anti-amphiphysin I antibody serum samples could be identified by immunohistochemistry on human cerebellum. In rat cerebellar sections evaluated by the avidin-biotin immunoperoxidase technique, the presence of anti-amphiphysin I antibodies was shown in only one serum sample, which corresponded to that with the strongest immunoreactivity in immunoblots. However, when the nine serum samples were evaluted by indirect immunofluorescence using a higher concentration, ${ }^{2}$ all but one gave an immunoreactive pattern compatible with antiamphiphysin I antibodies.

\section{CLINICAL-IMMUNOLOGICAL CORRELATION}

Seven anti-amphiphysin I antibody positive patients had $\operatorname{PND}(2.9 \%)$ and two had SCLC without neurological disorders (1.4\%). Six of the seven patients with PND had SCLC that was diagnosed after onset of the neurological symptoms. In one patient, who also was anti-Hu-antibody positive, the tumour could not be found at necropsy. We include this patient in the PND group because the patient presented typical clinical and neuropathological features of PEM/SN with neuronal loss and inflammatory infiltrates in the dorsal root ganglia and spinal cord. None of the patients without PND or with PND associated with anti-Yo, $\mathrm{Ri}$, or $\mathrm{Tr}$ antibodies had anti-amphiphysin I antibodies.

The clinical features of the seven patients with PND and anti-amphiphysin I antibodies are summarised in the table. Five of the seven patients developed symptoms of PEM/SN and four of them also had high titres of anti-Huantibodies. The other two patients had paraneoplastic cerebellar degeneration and 
Clinical features of anti-amphiphysin positive patients with paraneoplastic neurological disorders

\begin{tabular}{|c|c|c|c|c|c|c|}
\hline Patient & Age/sex & Clinical syndrome & $H u-A b$ & $\begin{array}{l}\text { Time (months) } \\
P N D / \text { tumour }\end{array}$ & Cancer & Outcome \\
\hline 1 & $60 / \mathrm{F}$ & Sensorimotor neuropathy & Yes & -23 & SCLC & Necropsy: PEM/SN† \\
\hline 2 & $58 / \mathrm{M}$ & $\mathrm{PEM} / \mathrm{SN}$ & Yes & -7 & SCLC & Necropsy: PEM/SN† \\
\hline 3 & $71 / \mathrm{M}$ & $\mathrm{PEM} / \mathrm{SN}$ and LEMS & Yes & -17 & SCLC & Necropsy: PEM/SN† \\
\hline 4 & $64 / \mathrm{M}$ & Sensory neuropathy & $\mathrm{No}^{\star}$ & 0 & SCLC & Death: pulmonary embolus \\
\hline 5 & $64 / M$ & Cerebellar syndrome & No & -18 & SCLC & Alive for 7 years \\
\hline 6 & $74 / \mathrm{M}$ & Opsoclonus/myoclonus & $\mathrm{No}^{\star}$ & -1 & SCLC & $\begin{array}{l}\text { Necropsy: perivascular/meningeal } \\
\text { inflammatory infiltrates. Moderate neuronal } \\
\text { loss in dentate nucleus and Purkinje cells }\end{array}$ \\
\hline 7 & $80 / \mathrm{F}$ & Sensory neuropathy & Yes & n.a. & None & 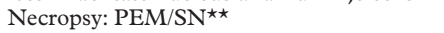 \\
\hline
\end{tabular}

$\mathrm{PEM} / \mathrm{SN}=$ Paraneoplastic encephalomyelitis/sensory neuronopathy; LEMS=Lambert-Eaton myasthenic syndrome; SCLC=small cell lung carcinoma; $\mathrm{NA}=$ not applicable.

${ }^{\star}$ Patients had low titre of $\mathrm{Hu}-\mathrm{Ab}$ similar to that found in $16 \%$ of SCLC without paraneoplastic neurological disorders. ${ }^{11}$

†Necropsy findings typical of PEM/SN with multifocal inflammatory infiltrates, reactive gliosis, and neuronal loss.

opsoclonus/myoclonus syndrome. The frequency of anti-amphiphysin I antibodies in patients with PND and SCLC (3.9\%) with $(2.7 \%)$ or without $(6.8 \%)$ anti-Hu-antibodies was not statistically different from that in patients with SCLC without PND.

\section{Discussion}

The main finding of the present study is that the frequency of anti-amphiphysin I antibodies was low in patients with SCLC and PND. The group of patients with the highest frequency of anti-amphiphysin I antibodies (6.8\%) corresponded with those with SCLC, PND, and negative anti-Hu-antibodies. However, even for this group the frequency of anti-amphiphysin I antibodies was not significantly different from that in patients with SCLC without PND.

The clinical range of anti-amphiphysin I antibody positive patients was not uniform. None developed clinical or neurophysiological features of SMS, five patients had PEM/SN and two developed other PND (see below). Four out of the five PEM/SN patients had anti-Hu-antibodies, a not surprising finding considering that the great majority of patients with $\mathrm{PEM} / \mathrm{SN}, 95 \%$ in this series, had anti-Hu-antibodies. ${ }^{6}$ Necropsy findings of the four patients with PEM/SN harbouring both anti-Hu-antibodies and anti-amphiphysin I antibodies were similar to those reported in anti-Hu-antibody positive patients with $\mathrm{PEM} / \mathrm{SN}$ but without anti-amphiphysin I antibodies. ${ }^{7}$ Although postmortem of one of our anti-amphiphysin I antibody positive patients did not show a tumour, this patient had anti-Hu-antibodies and typical clinical and postmortem features of PEM/SN raising the possibility that a small, tumour was overlooked.

The other two patients presented paraneoplastic cerebellar degeneration and the opsoclonus/myoclonus syndrome respectively. Patients with paraneoplastic cerebellar degeneration and SCLC, who do not develop other features of PEM/SN, usually lack anti-Huantibodies but $36 \%$ of them harbour P/Q type voltage gated calcium channel antibodies probably related to a higher than expected association with the Lambert-Eaton myasthenic syndrome. ${ }^{12}$ Similarly, anti-Huantibodies are rarely identified in paraneoplastic opsoclonus-myoclonus associated with SCLC although a few patients have been reported. ${ }^{13}{ }^{14}$ Excluding these exceptions, when paraneoplastic cerebellar degeneration and opsoclonus/myoclonus develop as isolated syndromes in patients with SCLC, they are not generally associated with any specific antineuronal antibody. Our series supports this notion, because anti-amphiphysin I antibodies were identified in only 3\% of paraneoplastic cerebellar degeneration and $14 \%$ of patients with opsoclonus-myoclonus and SCLC.

The low frequency of anti-amphiphysin I antibodies in patients with SCLC with PND, the lack of association with a particular neurological syndrome, and the similar frequency of anti-amphiphysin I antibodies in patients with SCLC without PND is by contrast with that described with anti-Hu-antibodies that are particularly associated with PEM/SN syndrome and SCLC. ${ }^{6}$ This is also by contrast with the close link between anti-amphiphysin I antibodies and SMS associated with breast cancer. $^{2}$

Our study suggests that the optimal immunohistochemical method to detect antineuronal antibodies depends on the type of antibody. In the current study, the use of recommended techniques and serum dilutions to detect antineuronal antibodies ${ }^{15}$ failed to identify anti-amphiphysin I antibodies which, however, were readily demonstrated using higher serum concentrations and a different protocol. ${ }^{2}$ In any case, immunoblot seems more sensitive than immunohistochemistry to detect antiamphiphysin I antibodies.

The anti-amphiphysin I antibodies from all our patients recognised fragment V (C terminus) of human amphiphysin I, although many of them also recognised additional fragments. This finding is in agreement with that previously reported in patients with SCLC with PEM/SN and patients with SMS and breast cancer. Interestingly, in a series of 30 mouse monoclonal antibodies which were raised against human amphiphysin I, only three were directed against fragment $\mathrm{V} .^{10}$ This indicates that the epitopes recognised by the human serum samples do not simply coincide with the most immunogenic portion of the molecule.

Anti-amphiphysin I antibodies may be useful in predicting the paraneoplastic origin of a neurological disorder in a given patient without known cancer because, in agreement with a previous report, ${ }^{5}$ we did not find anti-amphiphysin I antibodies in a large series of patients without PND but whose symptoms were initially suspected to be paraneoplastic. However, the low incidence of anti- 
amphiphysin I antibodies in patients with PND and SCLC represents a limitation for its use as a diagnostic marker of PND other than SMS.

We thank Dr M Solimena for many helpful discussions, Professor HM Meinck for providing one of the anti-amphiphysin positive serum samples, and Mercé Bonastre for her excellen technical assistance. The work was supported in part by
grantsSGR 9500027 Generalitat de Catalunya, FIS $97 / 2100$ grantsSGR 9500027 Generalitat de Catalunya, FIS 97/21
Madrid, Spain (FG), and NIH grant NS-26064, USA (JD).

1 David C, McPherson PS, Mundigl O, et al. The role of amphiphysin in synaptic vesicle endocytosis suggested by its binding to dynamin in nerve terminal. Proc Natl Acad Sci USA 1996;93:331-5.

2 Folli F, Solimena M, Cofiell R, et al. Autoantibodies to a 128 -kd synaptic protein in three women with the stiff-man syndrome and breast cancer. N Engl f Med 1993;328:546-$$
51
$$

3 De Camilli P, Thomas A, Cofiell R, et al. The synaptic vesicle-associated protein amphiphysin is the $128-\mathrm{kD}$ autoantigen of stiff-man syndrome with breast cancer. $\mathcal{F}$ Exp Med 1993;178:2219-3.

4 David C, Solimena M, De Camilli P. Autoimmunity in stiffman syndrome with breast cancer is targeted to the C-terminal region of human amphiphysin, a protein similar to the yeast proteins Rvs167 and Rvs161. FEBS Lett 1994; to the yeast

5 Dropcho EJ. Antiamphiphysin antibodies with small-cell lung carcinoma and paraneoplastic encephalomyelitis. Ann

6 Dalmau J, Graus F, Rosenblum MK, et al. Anti-Huassociated paraneoplastic encephalomyelitis/sensory neu- ronopathy. A clinical study of 71 patients. Medicine 1992;71:59-72.

7 Lennon VA, Manley HA, Kim K, et al. Amphiphysin autoantibodies: a paraneoplastic serological marker of breast and lung cancer-related encephalomyeloradiculoneuritides but not classical stiff-man syndrome [abstract]. Neurology 1997;48:A434.

8 Graus F, Dalmau J, Valldeoriola F, et al. Immunological characterization of a neuronal antibody (anti-Tr) associated with paraneoplastic cerebellar degeneration and Hodgkin's disease. ₹ Neuroimmunol 1997;74:55-61.

9 Saiz A, Arpa J, Sagasta A, et al. Autoantibodies to glutamic acid decarboxylase in three patients with cerebellar ataxia, late-onset insulin-dependent diabetes mellitus, and polyendocrine autoimmunity. Neurology 1997;49:1026-30.

10 Floyd S, Butler $\mathrm{MH}$, Cremona $\mathrm{O}$, et al. Expression of amphiphysin I, an autoantigen of paraneoplastic neurological syndromes, in breast cancer. Molecular Med 1998;4:2939 .

11 Graus F, Dalmau J, Reñé R, et al. Anti-Hu antibodies in patients with small-cell lung cancer: association with compatients with small-cell lung cancer: association with comOncol 1997;15:2866-72.

12 Mason WP, Graus F, Lang B, et al. Small-cell lung cancer, paraneoplastic cerebellar degeneration and the LambertEaton myasthenic syndrome. Brain 1997;120:1279-300.

13 Anderson NE, Budde-Steffen C, Rosenblum MK, et al. Opsoclonus, myoclonus, ataxia, and encephalopathy in adults with cancer: a distinct paraneoplastic syndrome. Medicine 1988;67:100-9.

14 Hersh B, Dangond F, Dalmau J, et al. Paraneoplastic opsoclonus-myoclonus associated with anti-Hu antibody. opsoclonus-myoclonus associ.
Neurology 1994;44:1754-5.

15 Moll JWB, Antoine JC, Brashear HR, et al. Guidelines on the detection of paraneoplastic anti-neuronal-specific antibodies. Neurology 1995;45:1937-41. 